\title{
Aplicação de métodos estatísticos na otimização da densidade de empacotamento de distribuições de pós de alumina
}

\section{(Optimization of the packing density of alumina powder distributions using statistical techniques)}

\author{
A. P. Silva ${ }^{1}$, A. M. Segadães ${ }^{2}$, T. C. Devezas ${ }^{1}$ \\ ${ }^{1}$ Departamento de Engenharia Electromecânica, Universidade Beira Interior \\ Covilhã, Portugal 6200-001 \\ abilio@dem.ubi.pt,tessalen@dem.ubi.pt \\ ${ }^{2}$ Departamento de Engenharia Cerâmica e do Vidro (CICECO) \\ Univ. Aveiro, Aveiro, Portugal 3810-193 \\ segadaes@cv.ua.pt
}

\begin{abstract}
Resumo
Fatores como a distribuição granulométrica e a morfologia condicionam as propriedades físicas de sistemas particulados (e.g. misturas de pós, pastas, concretos refratários), entre as quais a densidade de empacotamento. Podem ser encontrados, na literatura, vários modelos para a otimização da distribuição de tamanhos de partículas que maximiza o empacotamento, todos desenvolvidos para sistemas de esferas perfeitas (e.g. Furnas, Andreasen, Alfred). Neste trabalho, pós de alumina comercial (reativa e tabular) foram separados em nove frações granulométricas e, recorrendo a dois procedimentos distintos, construíram-se seis sistemas ternários diferentes e complementares de tamanhos de partículas. Usando a metodologia das superfícies de resposta e técnicas de análise estatística afins (programa de cálculo Statistica) obteve-se, em ambos os casos, a distribuição granulométrica que maximiza a densidade de empacotamento. Por comparação com as distribuições granulométricas teóricas, ficou demonstrada a validade do modelo teórico de Alfred para esferas perfeitas. Os resultados obtidos demonstram, assim, que o efeito prejudicial da não esfericidade das partículas pode ser, na realidade, compensado pela otimização da distribuição granulométrica global.

Palavras-chave: empacotamento de partículas, distribuição granulométrica, análise estatística.
\end{abstract}

Abstract

Particle size distribution and morphology are among the factors that affect the physical properties of particulate systems (e.g. powder mixtures, pastes, castable refractories). One such property is packing density. There are, in the literature, models that optimise particle size distributions for maximum packing density, all of them derived for spherical particles (e.g. Furnas, Andreasen, Alfred). In this work, commercial alumina powders (reactive and tabular) were divided into nine particle size classes. Following two different approaches, the latter were used to build six different ternary systems of complementary particle sizes. Using the response surface methodology and related statistical techniques (software Statistica), the particle size distribution that maximises the packing density was obtained in both cases and, by comparison with theoretical particle size distributions, the validity of Alfred's theoretical model for perfect spheres was demonstrated. These results clearly show that the harmful effect of the nonspherical shape of real particles can, in fact, be compensated by the optimization of the overall particle size distribution.

Keywords: particle packing, particle size distributions, statistical analysis.

\section{INTRODUÇÃO}

O processamento de pós cerâmicos com o objetivo de reduzir a porosidade e maximizar a densificação levou ao desenvolvimento, no final dos anos 20 e início de 30 do século $\mathrm{XX}$, dos princípios fundamentais do empacotamento de partículas. A abordagem deste problema por Furnas, Andreasen, Westman, Hugill, Talbot, Gaudin e Schuhmann, entre outros investigadores, mostrou claramente a influência da distribuição de tamanhos de partículas não só nas propriedades finais de corpos cerâmicos consolidados, como porosidade, densidade e resistência mecânica, como também nas características de trabalho durante o processamento de sistemas particulados, como viscosidade, escoabilidade, permeabilidade e velocidade de secagem $[1,2]$.

\section{Empacotamento de partículas}

Quando todas as partículas apresentam o mesmo tamanho (monotamanho), o volume intersticial mínimo é $26 \%$ do volume total (empacotamento em estrutura hexagonal compacta ou cúbica de faces centradas) e é independente do tamanho das partículas. Porém, os pós reais não se apresentam espontaneamente assim, mas antes nas mais variadas formas e 
segundo uma distribuição tendencialmente infinita de tamanhos de partículas, sem qualquer imposição de regularidade. Sendo assim, partículas de menor tamanho podem ocupar os interstícios deixados livres pelo empacotamento de partículas de tamanho superior, o que eleva a densidade do sistema. Portanto, a possibilidade de otimizar a distribuição de tamanhos de partículas, para maximizar o empacotamento e reduzir virtualmente a zero o volume intersticial, parece ser óbvia [1].

Esta é a base do modelo mais simples de empacotamento de partículas, proposto por Furnas, segundo o qual a eficiência de empacotamento máxima $\left(\mathrm{PE}_{\text {máx }}\right)$ de uma distribuição de partículas em três classes de tamanhos, grossos (L), médios (m) e finos (S), depende das eficiências de empacotamento das partículas de cada classe $\left(\mathrm{PE}_{\mathrm{L}}, \mathrm{PE}_{\mathrm{m}}\right.$ e $\mathrm{PE}_{\mathrm{S}}$, para as partículas da classe grossa, média e fina respectivamente), e é descrita pela equação (A):

$P E_{\text {máx }}=P E_{L}+\left(1-P E_{L}\right) \times P E_{m}+\left(1-P E_{L}\right) \times\left(1-P E_{m}\right) \times P E_{S}$

Se as eficiências de empacotamento das partículas em cada classe forem iguais, i.e. $\mathrm{PE}_{\mathrm{L}}=\mathrm{PE}_{\mathrm{m}}=\mathrm{PE}_{\mathrm{S}}=\mathrm{PE}$, então a eficiência de empacotamento máxima da distribuição ternária pode reescrever-se na forma da equação (B):

$P E_{\text {máx }}=P E+(1-P E) \times P E+(1-P E)^{2} \times P E$

A extrapolação desta relação para a mistura de uma infinidade de classes discretas de tamanhos, traduzirá a eficiência de empacotamento de uma distribuição contínua, que passa a ser descrita pela equação (C):

$C P F T=\frac{R^{\log D}-R^{\log D_{S}}}{R^{\log D_{L}}-R^{\log D_{S}}} \times 100$

Na equação (C), D é o tamanho da partícula, CPFT é a percentagem acumulada de partículas de diâmetro inferior a D (Cumulative Percent Finer Than), $D_{L}$ é o tamanho da maior partícula na distribuição, $\mathrm{D}_{\mathrm{S}}$ é o tamanho da menor partícula na distribuição, e $\mathrm{R}$ é a razão entre os volumes de partículas em classes de tamanhos adjacentes.

Para que se atinja um empacotamento ótimo devem, idealmente, ser garantidas razões entre tamanhos de 100:1, para eliminar o volume de vazios extra devido ao efeito de parede (na prática, procura-se trabalhar com razões de tamanhos entre 20:1 e 10:1 [3, 4]). McGeary [5] demonstrou, experimentalmente, que, num empacotamento de esferas de um só tamanho, se obtém uma densidade máxima entre 60 e 64\% e, para empacotamentos binários, é possível obter densidades de $85 \%$, desde que se garanta uma relação entre tamanhos de pelo menos sete. Para empacotamentos de sistemas ternários de esferas, McGeary obteve densidades de $90 \%$ e para sistemas quaternários chegou a valores superiores a $95 \%$.

A distribuição de monotamanhos de Furnas para empacotamento máximo não é natural, além de ser economicamente inviável e originar misturas de fraco desempenho [2].
Andreasen achava que a condição de semelhança subjacente ao empacotamento perfeito (comportamento fractal) deveria ser traduzida por uma lei de potência e, em alternativa ao modelo de Furnas, propôs a equação (D):

$C P F T=\left(\frac{D}{D_{L}}\right)^{q} \times 100$

Na equação (D), q é uma constante, designada por módulo da distribuição granulométrica. A representação gráfica da equação (D) em escala bi-logarítmica é uma recta de inclinação q. Para se obter um empacotamento máximo, o módulo da distribuição (q) deve estar compreendido entre um terço e um meio $(0,33<q<0,50)$ [1-3]. Utilizando os parâmetros de Andreasen, a densidade máxima obtida por McGeary [5] corresponde a um módulo de distribuição q=0,40.

A equação de Andreasen não contempla um tamanho mínimo de partícula finito e, no limite, esse mínimo é zero. Este fato pode parecer irrelevante pois, por exemplo, quando se calcula o tamanho médio das partículas, leva a um erro pouco significativo. Porém, quando se pretende obter, teoricamente, a área superficial específica ou a média do número de partículas, a contribuição das partículas menores é enorme (o número de partículas é inversamente proporcional ao cubo do seu tamanho, e a área superficial é inversamente proporcional ao quadrado do tamanho das partículas; logo, se o tamanho mínimo é zero, então a área específica e o número de partículas tendem para infinito). O mesmo tipo de efeito pode ser observado na curva cumulativa (CPFT) das distribuições granulométricas.

Como nas distribuições de tamanhos de partículas reais, existe sempre um tamanho mínimo, Dinger e Funk [3] introduziram, na equação de Andreasen, um tamanho finito para a menor partícula, o que levou ao modelo de Alfred descrito pela equação (E), que é conhecida como equação modificada de Andreasen:

$$
C P F T=\frac{D^{q}-D_{S}{ }^{q}}{D_{L}{ }^{q}-D_{S}{ }^{q}} \times 100
$$

Por simulação numérica utilizando a equação modificada de Andreasen, foi demonstrado que o expoente que optimiza a distribuição granulométrica para o empacotamento máximo de partículas esféricas é q=0,37 [3]. Na representação gráfica em escala bi-logarítmica, a curva CPFT de Alfred afasta-se da linha teórica de Andreasen e inflecte em direção a zero quando o tamanho de partícula se aproxima do tamanho mínimo presente na distribuição. As retas representativas do modelo de Andreasen são assimptotas para as curvas do modelo de Alfred.

Partindo dos modelos clássicos e da equação de Alfred, Zheng et al. [6], deduziram uma nova equação "de aperfeiçoamento" (equação (F)) e, por analogia com a equação modificada de Andreasen, explicitaram a constante de Dinger e Funk (equação $(\mathrm{G})$ ): 


$$
C P F T=\frac{D^{-\frac{\log \phi}{\log R}}-D_{S}^{-\frac{\log \phi}{\log R}}}{D_{L}^{-\frac{\log \phi}{\log R}}-D_{S}^{-\frac{\log \phi}{\log R}}}
$$$$
q=-\frac{\log \phi}{\log R}
$$

Nas equações (F) e $(G), \phi$ é a fração de vazios no empacotamento, ou fração intersticial, i.e. $\phi=1$-eficiência de empacotamento. A equação $(\mathrm{G})$ mostra que o módulo da distribuição q diminui com o aumento dos interstícios, ou seja, distribuições com módulo mais baixo terão também densidades de empacotamento mais baixas.

Todos estes modelos foram deduzidos para partículas esféricas e é óbvio que a não esfericidade perturba o empacotamento, diminuindo a sua eficiência. Quanto menos esféricas forem as partículas, menor será a densidade de empacotamento da distribuição, e quanto menor for o tamanho das partículas de forma irregular, maior será esse efeito. Aumentando a quantidade das partículas não esféricas, diminui a densidade e são afetadas todas as propriedades que com ela se relacionam. Assim, pode ser concluído que partículas de forma irregular podem originar valores de q inferiores ao limite mínimo de Andreasen (q=0,33) [6].

A Fig. 1 resume, em representação bi-logarítmica, os diferentes

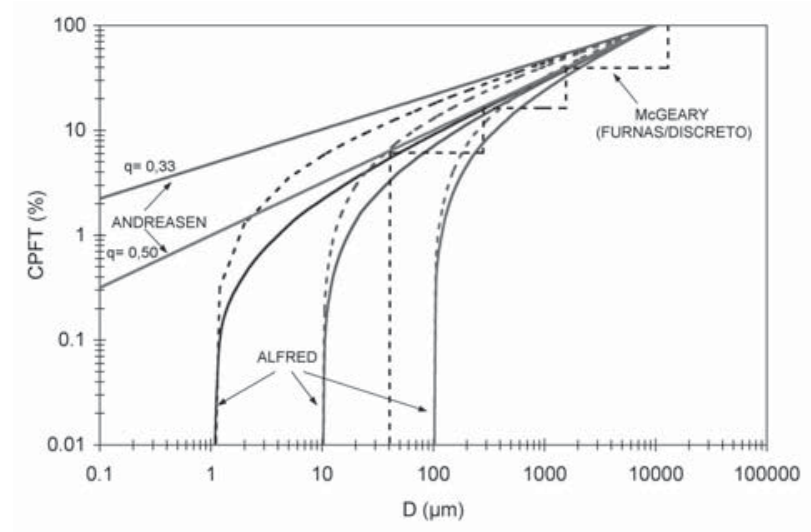

Figura 1: Representação gráfica dos modelos teóricos contínuos de Andreasen $\quad(q=0,33$ e $q=0,50)$ e de Alfred $(q=0,33$ e $q=0,50$, com $\mathrm{D}_{\text {min }}=1 \mathrm{~mm}, 10 \mu \mathrm{m}$ e $100 \mu \mathrm{m}$ ), e do modelo discreto de Furnas para um empacotamento quaternário preparado experimentalmente por McGeary [1].

[Figure 1: Graphical representation of theoretical models: for continuous distributions, after Andreasen ( $q=0.33$ and $q=0.50)$ and Alfred ( $q=0.33$ and $q=0.50$, for $D_{\min }=1 \mu \mathrm{m}, 10 \mu \mathrm{me} 100$ $\mu m)$; for a quaternary discrete distribution, after Furnas and as experimentally determined by McGeary [1].] modelos e suas condições limites [1]: o modelo contínuo de Andreasen para q=0,33 e q=0,50; o modelo contínuo de Alfred para os mesmos valores do módulo de distribuição mas com três tamanhos mínimos diferentes $\left(\mathrm{D}_{\text {mín }}=1 \mu \mathrm{m}, \mathrm{D}_{\text {mín }}=10 \mu \mathrm{m}\right.$ e $\mathrm{D}_{\text {min }}=100 \mu \mathrm{m}$ ); e o modelo de um empacotamento discreto de Furnas para quatro tamanhos de esferas perfeitas, que foi preparado experimentalmente por McGeary. Na representação gráfica, a equação $(\mathrm{F})$, chamada de "aperfeiçoamento", coincide com a equação de Alfred, mas a sua metodologia de aplicação prática é muito mais elaborada $[1,6]$.

Devido ao afastamento que se observa entre as representações gráficas dos modelos contínuos de Andreasen e de Alfred à medida que diminui o tamanho das partículas mais finas da distribuição, é fundamental que, quando essa faixa granulométrica for determinante no comportamento e controlo das propriedades da mistura de pós, seja adotado o modelo teórico que contenha a informação que lhe corresponde, não deixando de fora nenhuma "cauda" [7].

Para a otimização prática da distribuição granulométrica para maximizar a densidade de empacotamento [4], o modelo teórico preferido, por ser de aplicação mais simples e conduzir a resultados quase idênticos (mais eficiente, portanto) é o modelo de Andreasen, seguido do modelo de Alfred. Carniglia et al. [1] afirmam mesmo que a equação de Alfred é demasiado trabalhosa e "não há provas consistentes de que permita obter densidades de empacotamento mais elevadas que a equação de Andreasen". O modelo de Furnas é o que gera piores resultados (menos eficiente).

Recentemente, a Elkem ASA Materials [8] desenvolveu um programa de cálculo baseado nos modelos de Andreasen e de Alfred (o programa permite escolher um dos dois), a que chamou "Lisa", para a otimização das distribuições granulométricas de concretos refratários autoescoantes. Partindo das características dos pós em cada fração granulométrica disponível, o programa calcula a quantidade necessária de cada classe de partículas para se produzir uma mistura com um determinado módulo de distribuição. Utilizando um processo de convergência gráfica entre os pontos de sucessivas curvas de distribuição e a reta cujo declive é o módulo pretendido, obtém-se a melhor proporção das diferentes classes componentes da mistura e a correspondente curva final de distribuição [9].

\section{Análise Estatística}

A metodologia das superfícies de resposta e experiências com misturas [10-11] é um conjunto de técnicas estatísticas e matemáticas com importantes aplicações não só na criação, desenvolvimento e formulação de novos produtos, como também na otimização e melhoramento de produtos já existentes [12-18]. O estudo de misturas de diferentes componentes tem por objetivo encontrar um produto composto cujas características sejam superiores às características individuais de cada um dos componentes isoladamente.

A propriedade (resposta) que se pretende estudar, é função de um conjunto de variáveis independentes. A forma matemática da função é desconhecida e pode ser bastante 
complexa. O objetivo desta metodologia é encontrar uma função aproximada simples (modelo) que seja aceitável quando comparada com a realidade. A função aproximada normalmente é um polinômio de grau baixo (linear, quadrático, cúbico ou cúbico especial), que caracteriza adequadamente a propriedade em uma pequena região do espaço definido pelas variáveis independentes. Para um modelo ser adequado, os erros cometidos devem ser mínimos. Estes erros incluem todos os efeitos não contabilizados, como os erros de medida, ruídos, efeitos de outras variáveis não consideradas, etc.. Do ponto de vista estatístico, os erros (resíduos) deverão ter uma distribuição normal (i.e. aleatória) com média zero e variância constante. A adequação do modelo à resposta verdadeira exige, portanto, a avaliação correta dos resíduos: a representação da probabilidade normal em função dos resíduos deve ser aproximadamente linear, e a representação da função resíduos em função da resposta prevista, deve ser aleatória e razoavelmente simétrica $[10,11,17]$.

Para aplicar esta metodologia ao empacotamento de partículas de pós, a propriedade cuja função resposta se pretende calcular será a densidade de empacotamento que, como se disse atrás, depende das quantidades das várias frações granulométricas usadas e dos tamanhos das partículas em cada uma dessas frações granulométricas. Fixado o tamanho das partículas (ou seja, quais as frações granulométricas que serão usadas), a densidade de empacotamento passa a depender exclusivamente das quantidades das frações na mistura de pós. Esta é a consideração básica para a aplicação da metodologia das superfícies de resposta e experiências com misturas. As frações granulométricas passam, assim, a ser os constituintes da mistura de pós (variáveis independentes $x_{i}$, que variam entre 0 e 1 e cuja soma é igual à unidade), em função dos quais será expressa a composição da mistura. A forma final do polinômio (superfície de resposta) é calculada por regressão a partir de valores da propriedade, obtidos experimentalmente, para misturas selecionadas. O número e a composição destas misturas são, normalmente, definidos por uma rede de pontos uniformemente espaçados (arranjo simplex). Os valores dos coeficientes numéricos do polinômio final não são únicos, e pode haver várias soluções (vários polinômios) que satisfaçam o nível de convergência estipulado, sempre com um erro inerente.

Em analogia com o procedimento de Furnas, esta metodologia de cálculo pode ser aplicada com o objetivo de maximizar a densidade de empacotamento de uma distribuição de partículas em três classes de tamanhos, grossos, médios e finos. Estas três classes (constituintes do sistema) definem um triângulo (geralmente equilátero) de composições, onde se representam todas as misturas possíveis do sistema. Obtida a equação de regressão e validado o modelo, uma estimativa da propriedade pode ser calculada para qualquer outra mistura dos mesmos componentes, sem necessidade, portanto, de determinação experimental.

\section{MATERIAIS E MÉTODOS}

Como matérias primas, foram usadas a alumina tabular T60 (Alcoa), de densidade média 3,5 g/ $\mathrm{cm}^{3}$ e tamanho de partícula inferior a $2 \mathrm{~mm}$ (partículas não esféricas), e a alumina reativa CT3000SG (Alcoa), de densidade média 3,91 g/ $\mathrm{cm}^{3}$, com partículas de tamanho médio $1,8 \mu \mathrm{m}$ e todas as partículas abaixo de $20 \mu \mathrm{m}$. A alumina tabular foi classificada em oito frações granulométricas por separação com uma bateria de peneiras Retsch, de acordo com a norma ISO 3310/1. A alumina reativa, "AR", foi usada tal como fornecida. Excetuando a fração mais grossa, as distribuições de tamanhos em todas as frações granulométricas foram obtidas com um analisador de partículas com módulo para pós secos (Coulter LS 200, LS Variable Speed Fluid Module Plus), com capacidade para analisar partículas com tamanhos entre 0,4 e $2000 \mu \mathrm{m}$. Maiores detalhes sobre a caracterização e morfologia dos pós podem ser encontrados em outro trabalho [9].

As nove classes de tamanhos foram associadas em dois grupos de três sistemas ternários e, em cada sistema ternário, as composições das misturas (combinações de três frações granulométricas) para determinação experimental da densidade de empacotamento, foram definidas utilizando um arranjo simplex de dez pontos, no qual cada constituinte é avaliado em seis níveis de composição $\left(x_{\mathrm{i}}=0,1 /{ }_{6}, 1 /{ }_{3}, 1 /{ }_{2}, 2 /{ }_{3}, 1\right)$, como se pode observar no diagrama ternário da Fig. 2.

Todas as misturas foram homogeneizadas a seco em moinho planetário a $50 \mathrm{rpm}$ durante três minutos e a $100 \mathrm{rpm}$, durante mais três minutos. A mistura foi depois colocada num cone em aço inoxidável com uma altura de $100 \mathrm{~mm}$, diâmetro médio da base de 126,5 mm, e um volume útil de $418 \mathrm{~cm}^{3}$. Foi utilizada vibração para a acomodação dos pós, mas evitando a sua segregação. A densidade de empacotamento foi calculada pelo quociente entre a massa da mistura e o volume útil do cone. Para cada sistema ternário, os valores de densidade de empacotamento obtidos experimentalmente foram usados para calcular (usando o programa de cálculo Statistica 5.5 (StatSoft,

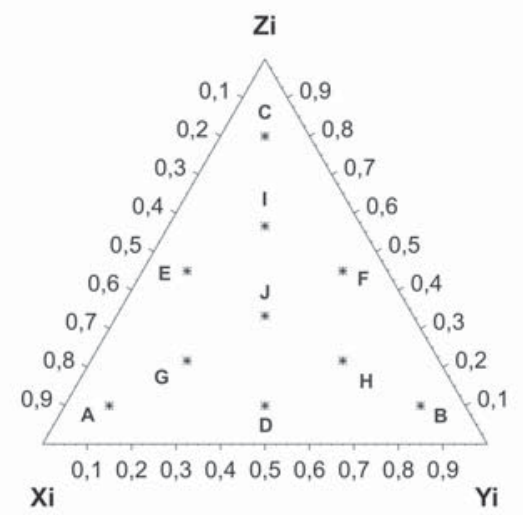

Figura 2: Diagrama triangular de composições, mostrando as dez misturas do arranjo simplex usado no cálculo da superfície de resposta.

[Figure 2: Composition triangular diagram, showing the ten simplex mixtures used in the calculation of the response surface.] 
Tabela I - Caracterização das classes granulométricas de alumina.

[Table I - Characterization of alumina particle size classes.]

\begin{tabular}{|c|c|c|c|c|c|c|c|c|}
\hline \multirow[t]{2}{*}{ Intervalos } & \multicolumn{3}{|c|}{ Valores teóricos } & \multicolumn{5}{|c|}{ Valores Experimentais } \\
\hline & Mín $[\mu \mathrm{m}]$ & $\operatorname{Med}[\mu \mathrm{m}]$ & Máx $[\mu \mathrm{m}]$ & $\mathrm{d}_{10}[\%]$ & $\mathrm{d}_{50}[\%]$ & $\mathrm{d}_{90}[\%]$ & $\begin{array}{c}\text { Média } \\
{[\mu \mathrm{m}]}\end{array}$ & $\begin{array}{c}\text { ASE } \\
{\left[\mathrm{cm}^{2} / \mathrm{g}\right]}\end{array}$ \\
\hline "AR" & 0,1 & 0,7 & 2,1 & 0,508 & 0,949 & 7,135 & 2,21 & 72700 \\
\hline “-500” & 0 & - & 25 & 3,004 & 11,64 & 25,98 & 13,26 & 2654 \\
\hline “-230” & 0 & - & 63 & 4,781 & 22,69 & 56,85 & 27,42 & 1771 \\
\hline “-200 +230" & 63 & - & 75 & 8,267 & 69,49 & 101,1 & 65,01 & 1004 \\
\hline$"-100+140 "$ & 106 & - & 150 & 94,48 & 145,2 & 208,3 & 146,6 & 333,4 \\
\hline$"-50+70 "$ & 212 & - & 300 & 239,4 & 333,4 & 517,8 & 377,7 & 51,89 \\
\hline “-30+40” & 425 & - & 600 & 468,9 & 667,9 & 1003 & 708,9 & 26,37 \\
\hline “-16 +20” & 850 & - & 1180 & 818,1 & 1127 & 1523 & 1148 & 15,9 \\
\hline$"-8+16 "$ & 1180 & - & 2360 & - & - & - & - & - \\
\hline
\end{tabular}

Tabela II - Composição (\% peso de cada uma das classes granulométricas constituintes) da mistura com a densidade de empacotamento máxima, em cada sistema ternário (mistura ótima).

[Table II : Composition (wt.\% of each constituent particle size class) of the mixture with maximum packing density in each ternary system (optimum mixture).]

\begin{tabular}{|c|c|c|c|}
\hline \multirow{3}{*}{$\begin{array}{c}\text { Sistema } \\
\text { ternário } \\
\text { constituintes }\end{array}$} & \multirow{3}{*}{$\begin{array}{c}\text { Intervalos } \\
\text { granulométricos }\end{array}$} & \multicolumn{2}{|c|}{ Composição da mistura ótima (\% peso) } \\
\hline & & constituintes & sistema otimizado \\
\hline & & individuais & \\
\hline \multirow[t]{3}{*}{ M2 } & $"-8+16 "$ & 36,3 & $>30 \%$ \\
\hline & “-50+70” & 36,3 & "M2 Optm" \\
\hline & “-230” & 27,5 & \\
\hline \multirow[t]{3}{*}{ M0 } & $“-16+20 ”$ & 47,3 & $>35 \%$ \\
\hline & $"-100+140 ”$ & 27,5 & "M0 Optm" \\
\hline & “-500” & 25,2 & \\
\hline \multirow[t]{3}{*}{ M1 } & “-30+40” & 50,5 & $17,5 \%$ \\
\hline & $“-200+230 ”$ & 39,5 & "M1 Optm” \\
\hline & "AR" & 10,0 & \\
\hline \multirow[t]{3}{*}{ Grossos } & $"-8+16 "$ & 45,00 & $56,25 \%$ \\
\hline & $"-16+20 "$ & 10,00 & "Grossos Optm” \\
\hline & $"-30+40 "$ & 45,00 & \\
\hline \multirow[t]{3}{*}{ Médios } & $“-50+70 ”$ & 45,00 & $25,00 \%$ \\
\hline & $"-100+140 "$ & 14,10 & “Médios Optm” \\
\hline & “-200 +230”, & 40,90 & \\
\hline \multirow[t]{3}{*}{ Finos } & "-230" & 37,22 & $18,75 \%$ \\
\hline & “-500” & 45,00 & "Finos Optm" \\
\hline & "AR" & 17,78 & \\
\hline
\end{tabular}


2000), módulo "Mixtures Designs") os coeficientes das equações de regressão que relacionam a densidade de empacotamento com as proporções das três frações granulométricas usadas. Em todos os casos foram testados os modelos linear, quadrático, cúbico e cúbico especial [10, 11, 17], tendo sido escolhido o modelo estatisticamente significante ao nível de $95 \%$.

A fiabilidade de cada uma das superfícies resposta foi garantida através da análise de variância, com o controlo da admissibilidade dos erros cometidos (desvios quadrados), da análise dos coeficientes de determinação e dos diversos modelos de adequação dos resíduos [11]. Em cada um dos sistemas, foram aleatoriamente escolhidas três misturas para validação experimental da resposta obtida. Estas misturas foram preparadas e caracterizadas tal como descrito para todas as outras.

Uma vez obtida uma superfície de resposta válida em cada um dos seis sistemas ternários, foi identificada a mistura de densidade de empacotamento máxima (mistura ótima) em cada sistema. As seis misturas ótimas foram associadas em dois novos sistemas ternários e foram calculadas, usando a mesma metodologia, as correspondentes superfícies de resposta. A mistura ótima de cada um destes dois sistemas otimizados foi então desdobrada nos correspondentes teores das nove classes de tamanhos originais que, juntamente com as respectivas distribuições granulométricas, foram introduzidos no programa de cálculo "Lisa", para o cálculo da curva de distribuição granulométrica global da mistura e do módulo de distribuição correspondente [9].
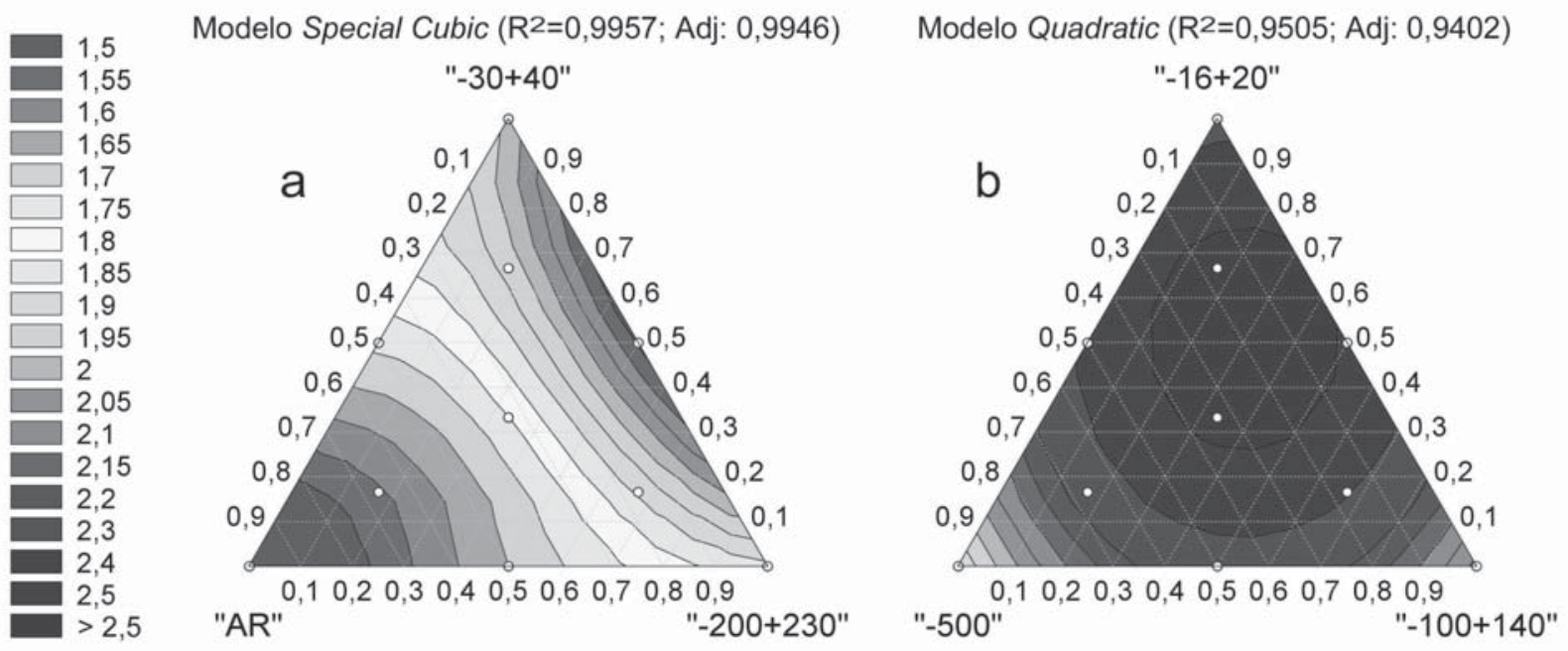

Modelo Special Cubic (R2=0,9629; Adj: 0,9533
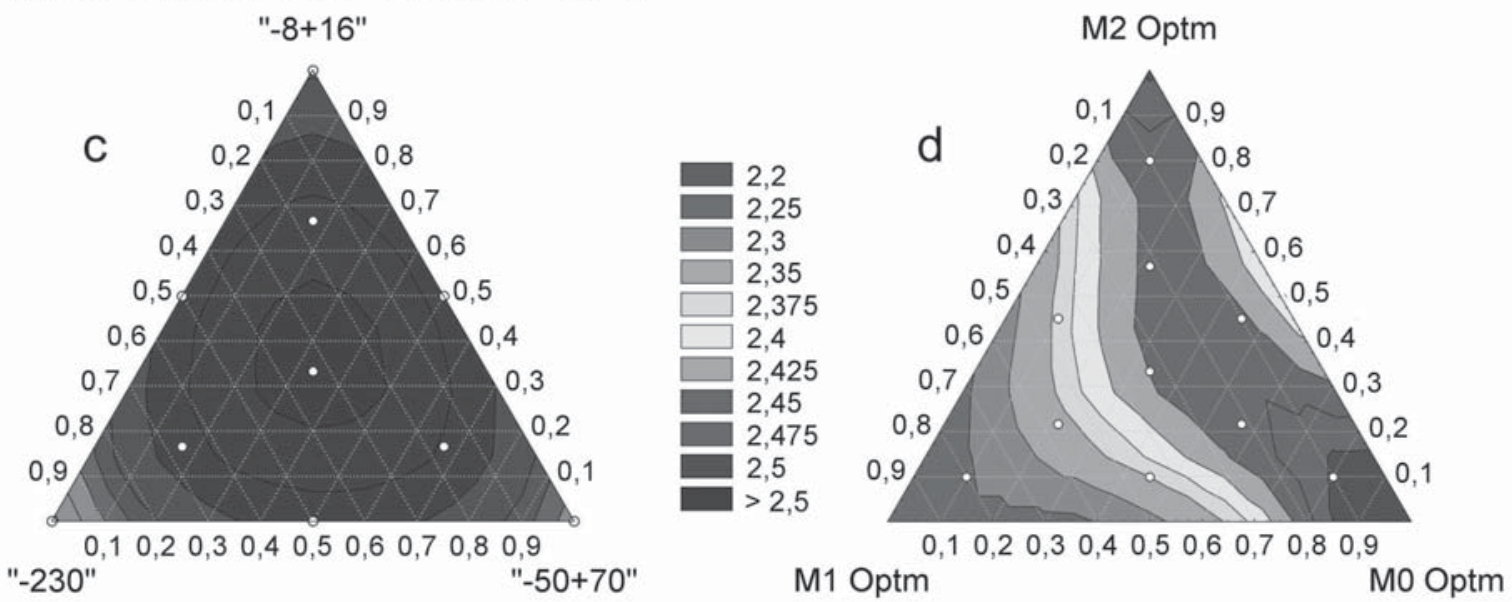

Figura 3: Superfícies de resposta da densidade de empacotamento nos sistemas: a) M1 [“AR", “-200+230”, “-30+40”]; b) M0 [“-500”, “$100+140 ", "-16+20 "]$; c) M2 [“-230", “-50+70", “-8+16”]; e d) sistema das misturas ótimas ["M1 Optm", "M0 Optm”, "M2 Optm”]. [Figure 3: Packing density response surfaces in the systems: a) M1 [“AR”, “-200+230”, “-30+40”]; b) M0 [“-500”, “-100+140, “16+20"]; c) M2 ["-230”, “-50+70”, “-8+16”]; and d) system of the optimum mixtures ["M1 Optm”, "M0 Optm”, "M2 Optm”].] 


\section{RESULTADOS E DISCUSSÃO}

A Tabela I mostra os parâmetros que caracterizam cada uma das nove frações granulométricas em que foram classificadas as matérias primas. A designação segue a numeração mesh (ASTM E 11-87): por exemplo, no intervalo “-50+70" o pó passou pela malha de 50 mesh e ficou retido na malha de 70 mesh [1]. Estes resultados mostram a disponibilidade de classes com tamanhos de partículas de 1 $\mu \mathrm{m}$ a $2 \mathrm{~mm}$, que permitem a construção de uma distribuição granulométrica contínua.

As nove classes de tamanhos foram associadas em sistemas ternários, da seguinte forma (ver Tabela II): i) Misturas de um intervalo fino com um médio e um grosso, designadas, respectivamente, por M1 ("AR", “-200+230” e "-30+40”), M0 (“-500”, “-100+140” e “-16+20”), e M2 (“-230”, “-50+70” e " $-8+16 ")$; ii) Misturas de três intervalos de tamanhos finos ("AR", “-500" e "-230"), três intervalos de tamanhos médios ("-200+230", $-100+140 "$ e "-50+70"), e três intervalos de tamanhos grossos (“-30+40”, “-16+20" e “-8+16”), designadas, respectivamente, por "Finos", "Médios" e "Grossos". Em cada um destes seis sistemas ternários foi determinada a densidade de empacotamento das nove misturas do arranjo simplex (Fig. 2) e calculada a superfície de resposta.

A Fig. 3 apresenta as superfícies de resposta para a densidade de empacotamento das misturas dos sistemas M1, M0 e M2, tal como calculadas com o Statistica e após análise
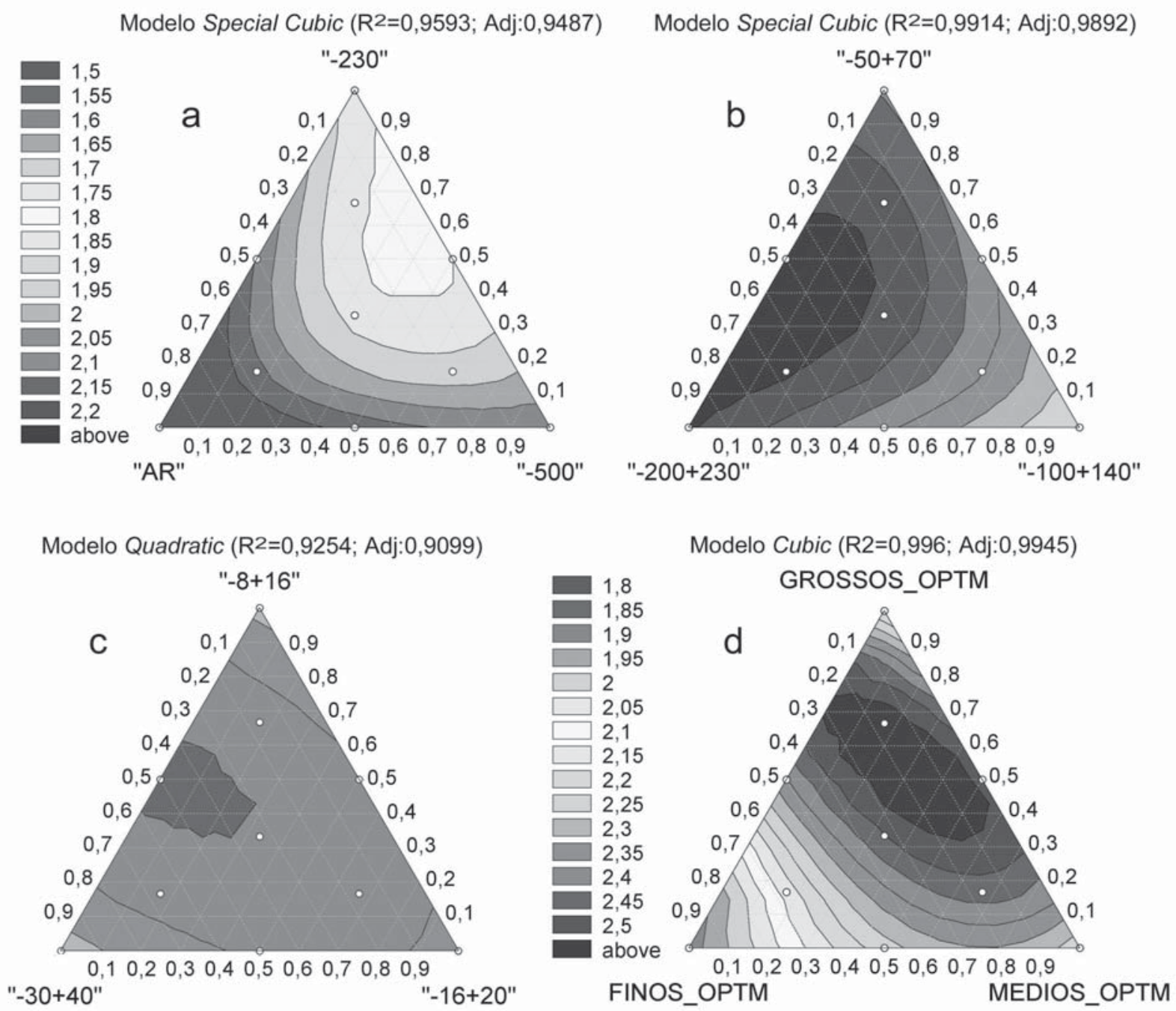

Figura 4: Superfícies de resposta da densidade de empacotamento nos sistemas: a) Finos [“AR", “-500”, “-230”]; b) Médios [“-200+230”, “-100+140", “-50+70”]; c) Grossos [“-30+40", “-16+20”, “-8+16”]; e d) sistema das misturas ótimas ["Finos Optm”, "Médios Optm”, "Grossos Optm"].

[Figure 4: Packing density response surfaces in the systems: a) Fine ["AR”, “-500”, “-230”]; b) Medium [“-200+230”, “-100+140”, “-50+70”]; c) Coarse ["-30+40", “-16+20”, “-8+16”]; and d) system of the optimum mixtures ["Fine Optm”, "Medium Optm”, "Coarse Optm"].] 

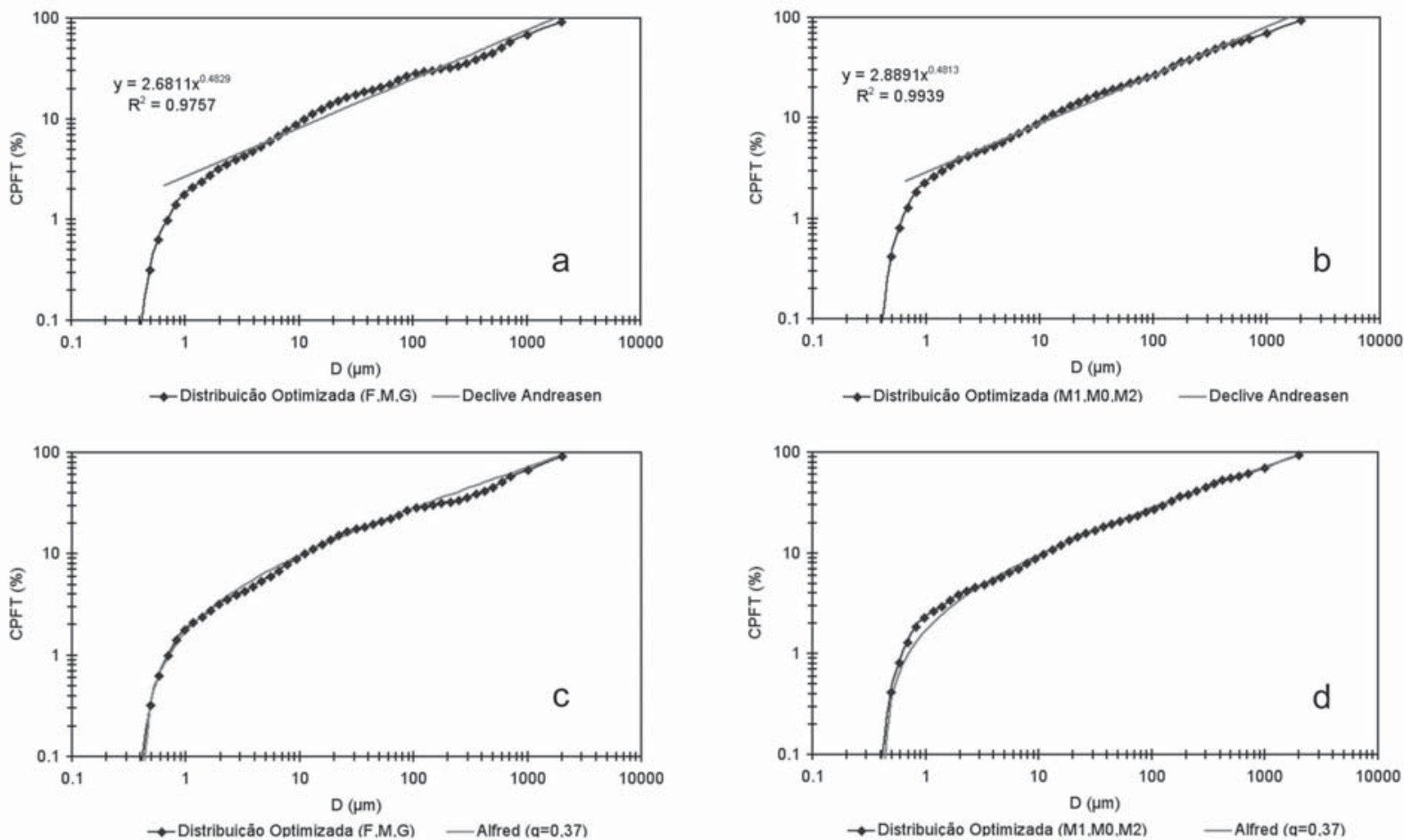

Figura 5: Comparação dos modelos teóricos de Andreasen (a, b) e de Alfred (c, d) com as curvas de distribuição granulométrica da mistura de densidade de empacotamento máxima nos sistemas Finos - Médios - Grossos (a, c) e M1 - M0 - M2 (b, d).

[Figure 5: Comparison of Andreasen's $(a, b)$ and Alfred's theoretical models $(c, d)$ with the particle size distribution curves for the maximum packing density mixture in the systems Fine - Medium - Coarse $(a, c)$ and $M 1-M 0-M 2(b, d)$.

dos erros e validação do modelo.

Da análise da resposta do sistema M1 (Fig. 3a), verifica-se que a alumina fina "AR" limita a densidade, devendo o seu teor na mistura de pós ser minimizado; pelo contrario, nos sistemas M0 e M2 (Figs. 3b e 3c) existem regiões interiores onde a densidade das misturas é francamente superior às dos constituintes. A Tabela II apresenta a composição, em percentagem em peso das classes constituintes (vértices do triângulo) da mistura que maximiza a densidade de empacotamento (mistura ótima), em cada um dos sistemas ternários.

A partir desta informação, foi construído um novo sistema tendo como constituintes as composições ótimas dos sistemas M1, M0 e M2 (sistema otimizado "M1 Optm" - "M0 Optm" - "M2 Optm"). O procedimento já descrito foi repetido para determinar a superfície de resposta da densidade de empacotamento no sistema otimizado, que está representada na Fig. 3d. Este sistema também apresenta uma gama de composições interiores onde a densidade de empacotamento é máxima. Essa região é relativamente independente dos teores de médios e grossos (estende-se quase paralelamente a esse lado do triângulo), com teores baixos do componente mais fino da mistura (entre 10 e $25 \%$ de "M1 Optm"), que parece ser o constituinte condicionante do empacotamento. A Tabela II indica a composição da mistura com densidade máxima de empacotamento no sistema otimizado.
Paralelamente, e seguindo um procedimento análogo, obtiveram-se as superfícies de resposta para a densidade de empacotamento nos sistemas "Finos", "Médios" e "Grossos" (Tabela II), representadas na Fig. 4.

Da análise destas respostas verifica-se que, para o sistema "Finos" (Fig. 4a), a densidade aumenta com a diminuição do teor de "AR", enquanto que no sistema "Médios" (Figura 4b) a densidade aumenta com a diminuição do teor de "-100 +140", e no sistema "Grossos" (Fig. 4c) a densidade aumenta com a redução do teor do intervalo, "-16 +20". A densidade de empacotamento atingida é mais baixa no sistema "Finos", onde se observa o menor valor de máximo $\left(1,5 \mathrm{~g} / \mathrm{cm}^{3}\right)$, e o máximo mais elevado é atingido no sistema "Médios" (superior a 2,2 g/cm³).

Em cada um dos sistemas "Finos", "Médios" e "Grossos" foram igualmente determinadas as composições das misturas ótimas, com densidade de empacotamento máxima (valores indicados na Tabela II). Usando como constituintes as composições otimizadas nos sistemas anteriores, foi construído um novo sistema otimizado ("Finos Optm" - "Médios Optm" - "Grossos Optm"), obtendo-se uma nova resposta (Fig. 4d) que permite avaliar a influência de cada uma das misturas otimizadas no sistema global. Nesta resposta há também uma região onde o empacotamento é máximo, cuja composição (teor das misturas ótimas) também é indicada na Tabela II. Mais uma vez se verifica que a mistura "Finos Optm" é a que parece 
condicionar mais o empacotamento. Para teores desta mistura superiores a $40 \%$ o valor da densidade diminui drasticamente.

As composições das duas misturas otimizadas foram então desdobradas nos correspondentes teores das nove classes de tamanhos originais que, juntamente com as respectivas distribuições granulométricas, foram introduzidos no programa de cálculo "Lisa", para o cálculo da curva de distribuição granulométrica global da mistura e do módulo de distribuição correspondente [9]. A curva da distribuição global da mistura otimizada obtida partindo do sistema "Finos" - "Médios" "Grossos" está representada nas Figs. 5a e 5c. E a curva da distribuição global da mistura otimizada obtida partindo do sistema M1 - M0 - M2 está representada nas Figs. 5b e 5d. As Figs. 5a e 5b comparam estas distribuições com o modelo de Andreasen; as Figs. 5c e 5d comparam estas distribuições com o modelo de Alfred.

De acordo com o modelo de Andreasen, ambas as distribuições apresentam um módulo q= 0,48 (Figs. 5a e 5b). Segundo o modelo de Alfred (Figs. 5c e 5d), ambas as distribuições se aproximam bastante da curva teórica que promove o empacotamento máximo das nove frações granulométricas usadas (representação da equação (E) com $\mathrm{q}=0,37$ imposto) [3].

No caso da distribuição otimizada a partir do sistema "Finos" - "Médios" - "Grossos" (Fig. 5c), o afastamento da curva experimental relativamente à curva teórica de Alfred de $\mathrm{q}=0,37$, é sempre inferior a $5 \%$, exceto no intervalo de tamanhos entre 209 e $592 \mu \mathrm{m}$, onde atinge $8,9 \%$. A curva experimental encontra-se mais próxima da curva teórica de Alfred de q=0,40. Relativamente à curva de $q=0,40,0$ afastamento máximo ocorre também entre os tamanhos 209 e $592 \mu \mathrm{m}$, com um desvio por defeito de $4,6 \%$.

Pela Fig. 5d, observa-se que as maiores diferenças (em valor absoluto) entre a distribuição otimizada a partir do sistema M1 - M0 - M2 e a curva teórica de Alfred com q=0,37, ocorrem em torno de $1000 \mu \mathrm{m}$, com um desvio por defeito de $2,3 \%$, e de $418 \mu \mathrm{m}$, com um desvio por excesso de $2,25 \%$. O afastamento observado no início do joelho da curva (tamanhos próximos de $1 \mu \mathrm{m}$ ), em valor absoluto é, na verdade, inferior a $0,6 \%$, i.e. trata-se de um efeito gráfico da escala logarítmica.

\section{CONCLUSÕES}

Partindo de pós comerciais de alumina separados em nove classes granulométricas e utilizando a metodologia de cálculo de superfícies de resposta e experiências com misturas, foi calculada a combinação ótima dessas classes que origina a máxima densidade de empacotamento da mistura de pós. Foram seguidos dois procedimentos experimentais independentes, que conduziram a resultados praticamente idênticos.

A análise das duas curvas de distribuição granulométrica finais à luz dos modelos teóricos de Andreasen e Alfred, desenvolvidos para misturas de esferas perfeitas, mostraram a validade do modelo de Alfred para o empacotamento de uma distribuição granulométrica real de pós de alumina (q=0,37). O efeito negativo da não esfericidade das partículas reais é, de acordo com estes resultados, compensado pela presença de uma infinidade de tamanhos na extensa distribuição completa final.

Já relativamente ao modelo de Andreasen, os resultados obtidos nos dois procedimentos correspondem a um módulo de distribuição global q=0,48 (próximo do valor máximo do intervalo $0,33<q<0,50$ ), e parecem contrariar os estudos [6] que indicam que misturas de partículas não esféricas devem ter valores baixos de módulo de distribuição (até mesmo q<0,33).

\section{REFERÊNCIAS}

[1] S. C. Carniglia, G. L. Barna, Handbook of Industrial Refractories Technology - Principles, Types, Properties and Applications, Reprint Edition, Noyes Publications, New York, USA (1992).

[2] S. Banerjee, Monolithic Refractories - A Comprehensive Handbook, The Am. Ceram. Soc. and World Scientific, Illinois, USA (1998).

[3] J. Funk, D. Dinger, Predictive Process Control of Crowded Particulate Suspensions - Applied to Ceramic Manufacturing, Kluwer Academic Plublishers, Boston, USA (1994).

[4] I. R. Oliveira, A. R. Studart, R. G. Pileggi, V. C. Pandolfelli, Dispersão e Empacotamento de Partículas - Princípios e Aplicações em Processamento Cerâmico, Fazendo Arte Editorial, S. Paulo (2000).

[5] R. K. McGeary, Mechanical Packing of Spherical Particles, J. Am. Ceram. Soc. 44, 10 (1961) 513-522.

[5] J. Zheng, P. Johnson, J. Reed, Improved Equation of the Continuous Particle Size Distribuition for Dense Packing, J. Am. Ceram. Soc. 73, 5 (1990) 1392-1398.

[7] D. R. Dinger (www.DingerCeramics.com), Ceramic Processing E-zine, 2, 4 (2004).

[8] T. Hanssen, Lisa Size Distribution Analyser, version 2.0, Elkem ITS \& Elkem Materials Refractories (www.material.elkem.com).

[9] A. P. Silva, A. M. Segadães, T. C. Devezas, Relações entre distribuição granulométrica, morfologia e empacotamento de partículas num sistema real: alta-alumina, Anais $47^{\circ}$ Cong. Bras. Ceram., J. Pessoa, PB (2003) 150-161.

[10] R. Myers, D. Montgomery, Response Surface Methodology, $2^{\text {nd }}$ Ed., John Wiley \& Sons, Inc., New York (2002).

[11] J. Cornell, Experiments with Mixtures, $3^{\text {rd }}$ Edition, John Wiley \& Sons, Inc., New York (2002).

[12] M. Jumaa, P. Kleinebudde, B. W. Muller, Mixture experiments with the oil phase of parenteral emulsions, Eur. J. Pharmac. Biopharmac. 46 (1998) 161-167.

[13] A. Gupta, Optimization of product performance of a paint formulation using a mixture experiment, J. Appl. Stat. 28, 2 (2001) 199-213.

[14] A. A. Zaman, F. Demir, E. Finch, Effects of process variables and their interactions on solubility of metal ions from crude kaolin particles: results of a statistical design of experiments, Appl. Clay Sci. 22 (2003) 237-250.

[15] M. Y. Noordin, V. C. Venkatesh, S. Sharif, S. Elting, A. Abdullah, Application of response surface methodology in 
describing the performance of coated carbide tools when turning AISI 1045 steel, J. Mater. Proces. Tech. 145 (2004) 46-58.

[16] S. L Correia, D. Hotza, A. M. Segadães, Simultaneous optimisation of linear firing shrinkage and water absorption of triaxial ceramic bodies using experiments design, Ceram. Int. 30, 6 (2004) 917-922.

[17] S. L Correia, K. A. S. Curto, D. Hotza, A. M. Segadães,
Using statistical techniques to model the flexural strength of dried triaxial ceramic bodies, J. Eur. Ceram. Soc. 24, 9 (2004) 2813-2818.

[18] S. L Correia, D. Hotza, A. M. Segadães, Otimização da resistência mecânica de corpos cerâmicos em função de matérias-primas e restrições de propriedades tecnológicas, Cerâmica (2004) submetido.

(Rec. 28/06/04, Ac. 24/09/04) 